\title{
Transverse interlayer order in lyotropic smectic liquid crystals
}

\author{
René van Roij, Peter Bolhuis, Bela Mulder, and Daan Frenkel \\ FOM-Institute for Atomic and Molecular Physics, Kruislaan 407, 1098 SJ Amsterdam, The Netherlands
}

(Received 9 January 1995)

\begin{abstract}
We present a theoretical and numerical study of the coupling of orientational and positional order in a smectic- $A$ phase of hard rods. Our density functional analysis suggests a strong spatial modulation of the orientation distribution function. Computer simulations confirm this prediction and show that the modulation is due to the presence of a finite concentration of particles positioned between smectic layers and oriented perpendicular to the director. This peculiar modulation of the orientational order should be experimentally observable in lyotropic smectic liquid crystals.
\end{abstract}

PACS number(s): $61.30 . \mathrm{Cz}, 64.70 . \mathrm{Md}, 61.20 . \mathrm{Ja}$

Onsager showed that excluded volume effects alone can lead to the formation of a liquid-crystalline phase. In his 1949 paper [1], he showed that a system of infinitely long hard rods exhibits a transition from an isotropic liquid $(I)$ to a nematic liquid crystal $(N)$. Subsequently, computer simulation studies have shown that rods of finite length also have an $I-N$ transition. Moreover, simulations have revealed that hard-core models can exhibit the more ordered smectic- $A$ $(\mathrm{Sm}-A)$ phase [2] and even, for oblate potentials, the columnar phase [3]. The richness of the phase diagram of hard rods inspired several authors to construct theories to explain this phase behavior. Such theories were developed, first for aligned rods [4-7] and later also for freely rotating rods $[8,9]$. In Refs. [4-9], the phase behavior of hard rods is discussed in the framework of density functional theory (DFT), in which the Helmholtz free energy $\mathscr{F}$ of the system is expressed as a functional of the one-particle distribution function $\rho(\vec{r}, \hat{\omega})$ [10]. Here $\vec{r}$ and $\hat{\omega}$ denote the position of the center of mass and the orientation of a rod, respectively. As the stable phase is the one with the lowest free energy, the aim of the DFT is to find a form for the one-particle distribution $\rho(\vec{r}, \hat{\omega})$ such that $\mathscr{F}$ is minimized while maintaining the normalization $\int d \vec{r} d \hat{\omega} \rho(\vec{r}, \hat{\omega})=N=n V$, where $V$ is the volume of the system, $N$ the total number of rods, and $n$ the number density. Although the DFT's for freely rotating rods are based on fairly crude approximations, they reproduce computer simulation results for the $I, N$, and $\mathrm{Sm}-A$ phase of rods rather well. However, an important approximation made in both Ref. [8] and Ref. [9] is the assumption that orientational and positional order are decoupled. This implies that $\mathscr{F}$ is minimized over the class of functions $\rho(\vec{r}, \hat{\omega})$ $=n \psi(\hat{\omega}) \Phi(\vec{r})$. In other words, the orientation distribution function (ODF) $\psi(\hat{\omega})$ is assumed to be position independent. This assumption is already suspect on purely physical grounds, since differences in local density should lead to differences in orientational order. Intuitively one would expect that the orientational order is less pronounced at low local density, i.e., in between the smectic layers. The importance of the coupling of orientational and positional order in the $\mathrm{Sm}-A$ phase is also manifest in molecular mean-field theories and in phenomenological Landau-de Gennes type theories, especially when the orientational order is not saturated at the $N-\mathrm{Sm}-A$ transition $[11-13]$. This is precisely the case in systems of sufficiently short freely rotating rods, where the $I-N$ coexistence region is close to the $N-\mathrm{Sm}-A$ transition. In this report we analyze the role of translationorientation coupling in the context of a simple DFT for hard rods which is able to describe $I, N$, and Sm- $A$ phases. We find that the coupling of orientational and positional order is very strong and results in an interesting feature of the Sm- $A$ phase. We also present computer simulations on a system of hard spherocylinders that support the theoretical analysis.

The simplest approximation for the free energy of a system of monodisperse hard rods is the virial expansion, truncated after the first non-ideal-gas term. In this so-called Onsager approximation $\mathscr{F}$ reads

$$
\begin{aligned}
\beta \mathscr{F}[\rho(\vec{r}, \hat{\omega})]= & \int d \vec{r} d \hat{\omega} \rho(\vec{r}, \hat{\omega})[\ln \rho(\vec{r}, \hat{\omega}) \mathscr{T}-1] \\
& -\frac{1}{2} \int d \vec{r} d \hat{\omega} d \vec{r}^{\prime} d \hat{\omega}^{\prime} f_{M}\left(\vec{r}, \hat{\omega} ; \vec{r}^{\prime}, \hat{\omega}^{\prime}\right) \\
& \times \rho(\vec{r}, \hat{\omega}) \rho\left(\vec{r}^{\prime}, \hat{\omega}^{\prime}\right),
\end{aligned}
$$

where $\beta$ is the inverse temperature and $\mathscr{T}$ the de Broglie thermal "volume." The shape of the rod enters the theory via the Mayer function $f_{M}\left(\vec{r}, \hat{\omega} ; \vec{r}^{\prime}, \hat{\omega}^{\prime}\right)$, which in the case of hard particles equals -1 if the two particles overlap, and is zero otherwise. Minimizing $\mathscr{F}$ while maintaining the normalization of $\rho$ yields

$$
\begin{aligned}
\frac{\delta \beta \mathscr{F}}{\delta \rho(\vec{r}, \hat{\omega})}= & \ln \rho(\vec{r}, \hat{\omega}) \mathscr{V} \\
& -\int d \vec{r}^{\prime} d \hat{\omega}^{\prime} f_{M}\left(\vec{r}, \hat{\omega} ; \vec{r}^{\prime}, \hat{\omega}^{\prime}\right) \rho\left(\vec{r}^{\prime}, \hat{\omega}^{\prime}\right)=\beta \mu
\end{aligned}
$$

where the Lagrange multiplier $\mu$ is fixed by the condition that the total number of particles is $N$. Kayser and Raveché [14] have studied this nonlinear equation in the case of the spatially homogeneous $I$ and $N$ phases. Starting from the observation that the isotropic distribution $\rho_{i s o}=n / 4 \pi$ is a solution of (2), they linearized (2) about $\rho_{\text {iso }}$ and found solutions of nematic character in the resulting eigenvalue equation. The same kind of bifurcation analysis will be used here. 
The difference is that the reference solution is now the nematic distribution $\rho_{0}(\vec{r}, \hat{\omega})=n \psi_{0}(\hat{\omega})$, which from (2) satisfies

$$
\psi_{0}(\hat{\omega})=\frac{\exp \left[-n \int d \hat{\omega}^{\prime} E\left(\hat{\omega}, \hat{\omega}^{\prime}\right) \psi_{0}\left(\hat{\omega}^{\prime}\right)\right]}{\int d \hat{\omega} \exp \left[-n \int d \hat{\omega}^{\prime} E\left(\hat{\omega}, \hat{\omega}^{\prime}\right) \psi_{0}\left(\hat{\omega}^{\prime}\right)\right]},
$$

where $E\left(\hat{\omega}, \hat{\omega}^{\prime}\right)$ is the orientation dependent excluded volume. The self-consistency relation (3) for the nematic reference distribution can readily be solved numerically for a given density $n[15,16]$. Possible other solutions of (2) can be found by inserting $\rho(\vec{r}, \hat{\omega})=\rho_{0}(\vec{r}, \hat{\omega})+\varepsilon \rho_{1}(\vec{r}, \hat{\omega})$ with $\varepsilon \ll 1$ into (2) and solving order by order in $\varepsilon$. The linear term in $\varepsilon$ gives

$$
\begin{aligned}
\frac{\rho_{1}(\vec{r}, \hat{\omega})}{\rho_{0}(\vec{r}, \hat{\omega})}-\frac{1}{4 \pi V} \int d \vec{r} d \hat{\omega} \frac{\rho_{1}(\vec{r}, \hat{\omega})}{\rho_{0}(\vec{r}, \hat{\omega})} \\
\quad=\int d \vec{r}^{\prime} d \hat{\omega}^{\prime} f_{M}\left(\vec{r}, \hat{\omega} ; \vec{r}^{\prime}, \hat{\omega}^{\prime}\right) \rho_{1}\left(\vec{r}^{\prime}, \hat{\omega}^{\prime}\right),
\end{aligned}
$$

where we used the normalization condition $\int d \vec{r} d \hat{\omega} \rho_{1}(\vec{r}, \hat{\omega})$ $=0$. It is easily verified that Eq. (4) has "smectic" solutions $\rho_{1}(\vec{r}, \hat{\omega})=n \psi_{1}(\hat{\omega}) \cos (\vec{q} \cdot \vec{r}) \quad$ for arbitrary $\quad \vec{q} \neq 0$, where $\psi_{1}(\hat{\omega})$ satisfies the eigenvalue equation

$$
\psi_{1}(\hat{\omega})=n \psi_{0}(\hat{\omega}) \int d \hat{\omega}^{\prime} \hat{f}_{M}\left(\vec{q}, \hat{\omega}, \hat{\omega}^{\prime}\right) \psi_{1}\left(\hat{\omega}^{\prime}\right)
$$

Here $\hat{f}_{M}$ denotes the Fourier transform of $f_{M}$. In the case of the $N-\mathrm{Sm}-A$ bifurcation, we only need to consider $\vec{q}=q \hat{z}$, where $\hat{z}$ is the nematic director and the normal of the smectic layers. The full $N-\mathrm{Sm}-A$ bifurcation analysis consists of finding the smallest value $n=n^{*}$ [with the corresponding ODF $\psi_{0}=\psi_{0}^{*}$ according to Eq. (3)] which gives rise to a nontrivial solution $\psi_{1}=\psi_{1}^{*}$ of (5). The associated wave number $q=q^{*}$ is related to the smectic layer spacing $\lambda^{*}=2 \pi / q^{*}$ at the bifurcation.

We have performed the $N-\mathrm{Sm}-A$ bifurcation analysis in the case of freely rotating spherocylinders of total length $L+D$ and diameter $D$ for several values of the aspect ratio $\kappa=L / D$. Denoting the volume of a rod $v_{0}$ and the packing fraction $\eta=n v_{0}$, we found $0.841<\eta^{*}<1.256$ and $1.27>\lambda^{*} /(L+D)>1.20$, when $\kappa$ is decreased from 20 to 3.5. The nematic order parameter at the $N-\mathrm{Sm}-A$ bifurcation, $S^{*}=\int d \hat{\omega} P_{2}(\hat{\omega} \cdot \hat{z}) \psi_{0}^{*}(\hat{\omega})$, varied from 0.990 to 0.656 in the same $\kappa$ regime. These values are in good agreement with an asymptotic (large $\kappa$ ) analysis by Poniewierski [17]. The $N-\mathrm{Sm}-A$ bifurcation enters the $I-N$ coexistence region at $\kappa=3.450 \pm 0.001$. The surprising feature of the theory is related to the comparison of $\psi_{0}^{*}(\hat{\omega})$ and $\psi_{1}^{*}(\hat{\omega})$, which are assumed to be identical in the decoupling approximation (apart from a multiplicative constant which can be absorbed in $\varepsilon$ ). In Fig. 1 we have depicted $\psi_{0}^{*}(\hat{\omega})$ and $\psi_{1}^{*}(\hat{\omega})$ as a function of $\theta$, the angle between $\hat{\omega}$ and $\hat{z}$, for the aspect ratios $\kappa=3.5$ and $\kappa=10$. The inset shows the ratio $\chi_{1}^{*}(\hat{\omega}) \equiv \psi_{1}^{*}(\hat{\omega}) / \psi_{0}^{*}(\hat{\omega})$. For clarity we changed the normalizations such that $\psi_{0}^{*}(\hat{z})=\psi_{1}^{*}(\hat{z})=1$. For both values of $\kappa$

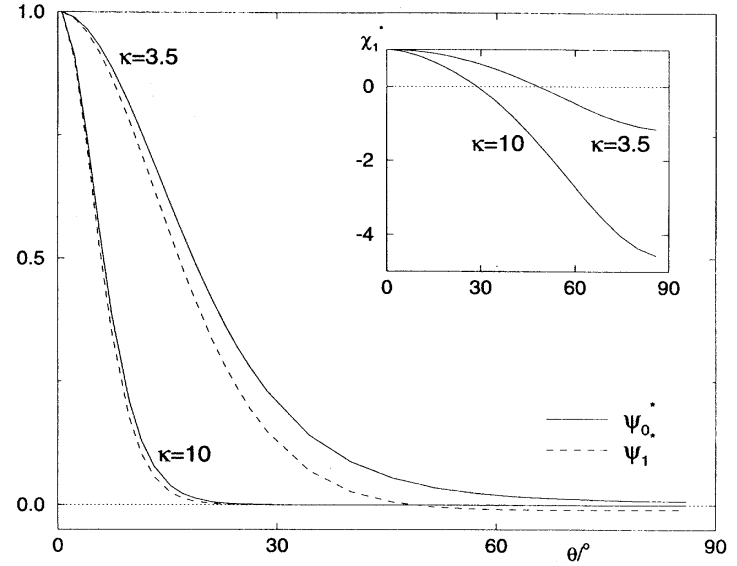

FIG. 1. Rescaled $\psi_{0}^{*}$ and $\psi_{1}^{*}$ for aspect ratios $\kappa=3.5$ and 10 as a function of $\theta$ (see text). The inset shows the ratio $\chi_{1}^{*} \equiv \psi_{1}^{*} / \psi_{0}^{*}$ for the same $\kappa$ values, from which the change of sign is clearly visible. The sign change is hardly relevant for $\kappa=10$, since it takes place when $\psi_{0}^{*}$ is vanishingly small. This is not the case for $\kappa=3.5$, indicating a coupling of orientational and positional order.

we not only see that $\psi_{0}^{*} \neq \psi_{1}^{*}$, but also that $\chi_{1}^{*}$ (and hence $\left.\psi_{1}^{*}\right)$ changes sign at a ( $\kappa$-dependent) critical angle $\theta=\theta_{c}$. Since for $\kappa=10$ the relative deviation between $\psi_{0}^{*}$ and $\psi_{1}^{*}$ is only appreciable when $\psi_{0}^{*}$ is vanishingly small, we conclude that the decoupling approximation is rather accurate in this case. This, however, is not true for $\kappa=3.5$, where the sign change of $\psi_{1}^{*}$ takes place when $\psi_{0}^{*}$ is still non-negligible. This sign change, then, presages a strong coupling of orientational and positional order. This can be understood, if one realizes that the one-particle distribution function in the vicinity of the $N-\mathrm{Sm}-A$ bifurcation reads

$$
\rho_{\mathrm{Sm}-A}(\vec{r}, \hat{\omega})=n^{*} \psi_{0}^{*}(\hat{\omega})\left[1+\varepsilon \chi_{1}^{*}(\hat{\omega}) \cos \left(q^{*} z\right)+O\left(\varepsilon^{2}\right)\right]
$$

Hence the sign change of $\chi_{1}^{*}$ at $\theta=\theta_{c}$ gives rise to a density modulation of rods oriented within the cone $0<\theta<\theta_{c}$, which is in antiphase with that of rods oriented out of this cone. This result implies not only the expected reduction of average orientational order between the layers compared to that in the layers, but it also suggests a specific mechanism for this reduction, namely an enhancement of the fraction of particles with an orientation transverse to the director, rather than, e.g., a broadening of the original single peaked nematic distribution. Given the typical geometry of the smectic phase with a layer spacing $\lambda /(L+D)=\alpha \approx 1.25$, it is plausible that particles in between the layers are either oriented along the director, bridging two layers, or oriented in a narrow cone of opening angle $\gamma \approx \arcsin (\alpha-1) \approx 14^{\circ}$ transverse to the director, leading to the surprising conclusion that the ODF in between the layers could in fact be bimodal. This unexpected mechanism, so strongly hinted at by the above analysis, is in fact confirmed by the computer simulation results presented below.

In order to be able to observe the modulation in antiphase of the transversely oriented particles, it is crucial that $\psi_{0}^{*}\left(\theta_{c}\right)$ is not vanishingly small. Experiments, or computer 

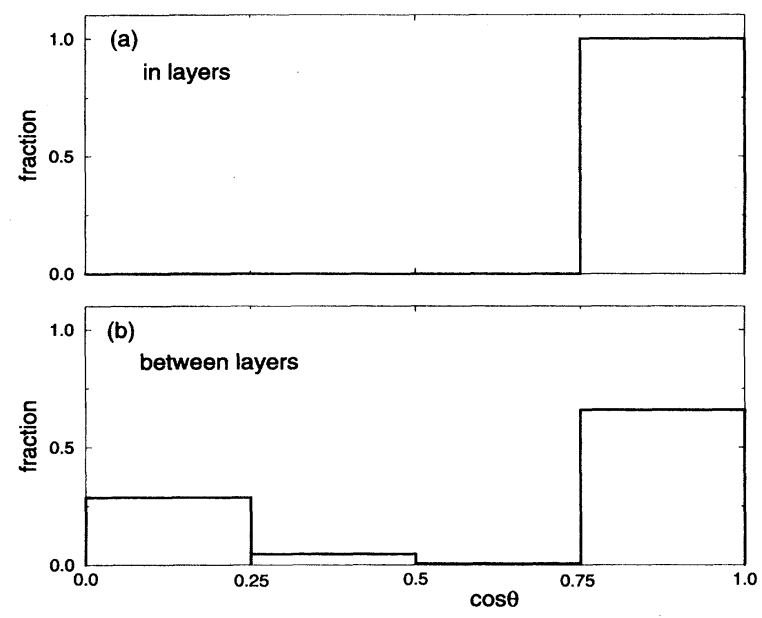

FIG. 2. Histograms of the local orientation distribution function, giving the local fraction of particles oriented in four intervals covering $0<\theta<90^{\circ}$. (a) represents the ODF in the smectic layers and (b) between the smectic layers. Note that equidistant $\cos \theta$ intervals correspond to $\theta$ intervals with equal solid angle area.

simulations, should therefore be carried out for short rods, where the $N-\mathrm{Sm}-A$ transition is close to the $I-N$ coexistence region. The unphysically high packing fractions, predicted by the Onsager functional applied to short rods, are not of great concern at this stage, since we are mainly interested in the generic coupling of orientational and positional order. Physical packing fractions can be obtained using, e.g., the Parsons-Lee density functional $[18,19]$, which includes this coupling at the same level as the Onsager functional.

In order to test our theoretical predictions, we carried out a computer simulation study of a system of hard spherocylinders with $\kappa=3.8$ at $\eta=0.527$. At this value of $\kappa$ we expect the $N-\mathrm{Sm}-A$ transition at $0.50<\eta<0.52$, [2] which is just above the $I-N$ coexistence at $0.47<\eta<0.49$. We prepared the smectic phase by aligning 540 spherocyclinders in 5 layers. The system was equilibrated using variable box-shape Monte Carlo simulation, in which the volume of the simulation box was fixed but its shape could vary in order to relax possible anisotropies in the stress tensor. Data were collected in a long molecular dynamics (MD) simulation of $2 \times 10^{4}$ collisions per particle.

We have measured the density-density correlation function $g(z)=\langle\rho(z) \rho(0)\rangle$ and the local nematic order parameter $S(z)$, where $\rho(z)=\int d x d y d \hat{\omega} \rho(\vec{r}, \hat{\omega})$ and $S(z)=\left\langle P_{2}(\cos \theta)\right\rangle(z)$. The angular brackets denote the average over the MD trajectory, $z$ denotes the spatial direction perpendicular to the smectic layers, and $\theta$ the angle between the particle orientation and the director. The $z$-dependent correlation functions were determined for $z$ intervals of thickness $0.25 D$. From the modulations of $g(z)$ with periodicity $\lambda=1.34 L$ we deduced that the number density between the layers is about a factor of 10 smaller than in the middle of the layers. We found similar modulations in $S(z)$, which varied smoothly from the value 0.894 in the middle of the smectic layers to the surprisingly low value of 0.052 in between the layers, confirming the predicted coupling of orientational and positional order. Histograms revealing the struc-

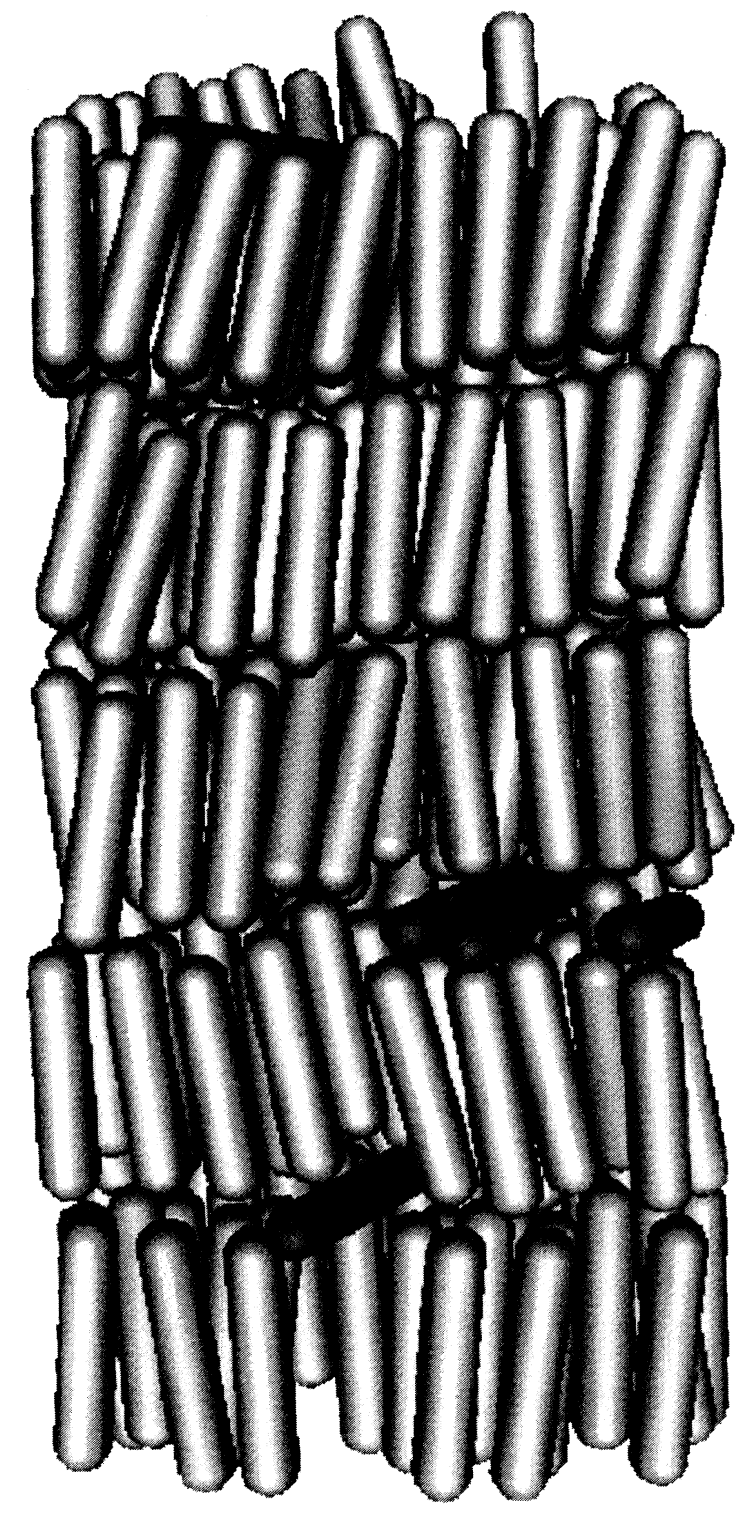

FIG. 3. Typical equilibrated configuration of the $\mathrm{Sm}-A$ phase of spherocylinders with $\kappa=3.8$ at $\eta=0.527$. We highlighted the transverse interlayer particles. The apparent alignment of these particles is accidental; statistically there is no preferred in-plane direction. From the ODF in Fig. 2(b) one would expect to see more "parallel" than "transverse" particles in between the layers in a single snapshot. The low density of particles in between the layers, however, prevented us from finding a single snapshot conveniently visualizing both possible orientations at the same time.

ture of the associated local ODF's are depicted in Fig. 2, where we have divided the interval $0<\cos \theta<1$ into four equidistant parts. Figure 2(a) represents the ODF in the middle of the smectic layers, which shows a single peaked (nematic) structure about $\theta=0$. Clearly, the particles positioned in the middle of the layers are all oriented in the cone $0<\theta<\arccos (0.75) \approx 41^{\circ}$. Figure 2 (b) represents the ODF in between the smectic layers. Indeed we see a strongly bimodal distribution with a second peak in the interval $\arccos (0.25) \approx 76^{\circ}<\theta<90^{\circ}$, confirming the presence of 
"transverse" particles in between the layers. This is visualized in Fig. 3, where a typical snapshot of a Sm- $A$ configuration of spherocylinders with $\kappa=3.8$ at $\eta=0.527$ is shown. Clearly, most of the particles point in the same direction and are positioned in layers. A finite fraction, however, points in a perpendicular direction and is positioned in between the layers, whereas virtually no particle has an orientation in between these two extremes. It should be stressed that this snapshot is obtained after equilibration of an initially perfectly aligned smectic configuration.

In summary, we have presented theoretical and numerical evidence for transverse interlayer order in the Sm- $A$ phase of short hard rods. The theoretical prediction, based on the simple Onsager free energy density functional, shows that the density of particles oriented within a cone $0<\theta<\theta_{c}$ tends to be modulated in antiphase with that of particles oriented out of the cone. It shows that a coupling of orientational and positional order is required in the theoretical description of short rods [20]. The numerical simulations reveal a non-negligible equilibrium concentration of particles positioned in between the layers and oriented perpendicular to the layers, giving rise to a bimodal local orientation distribution function in between the layers. We speculate that this surprising phenomenon should be observable experimentally, for instance by analysis of electron micrographs of the freeze-fractured $\mathrm{Sm}-A$ phase of rodlike viruses [23].

It is a pleasure to thank Peter Bladon and Joost Frenken for a critical reading of the manuscript. The work is part of the research program of the "Stichting voor Fundamenteel Onderzoek der Materie FOM," which is financially supported by the "Nederlandse Organisatie voor Wetenschappelijk Onderzoek (NWO)."
[1] L. Onsager, Ann. N.Y. Acad. Sci. 51, 627 (1949).

[2] J.A.C. Veerman and D. Frenkel, Phys. Rev. A 41, 3237 (1990).

[3] J.A.C. Veerman and D. Frenkel, Phys. Rev. A 45, 5632 (1992).

[4] B. Mulder, Phys. Rev. A 35, 3095 (1987).

[5] X. Wen and R.B. Meyer, Phys. Rev. Lett. 59, 1325 (1987).

[6] A.M. Somoza and P. Tarazona, Phys. Rev. Lett. 61, 2566 (1988).

[7] R. Holyst and A. Poniewierski, Mol. Phys. 71, 561 (1990).

[8] A. Poniewierski and R. Holyst, Phys. Rev. Lett. 61, 2461 (1988); Phys. Rev. A 41, 6871 (1990); A. Poniewierski and T.J. Sluckin, Phys. Rev. A 43, 6837 (1991).

[9] A.M. Somoza and P. Tarazona, Phys. Rev. A 41, 965 (1990).

[10] R. Evans, Adv. Phys. 28, 143 (1979).

[11] W.L. McMillan, Phys. Rev. A 4, 1238 (1971); 6, 936 (1972).

[12] R.B. Meyer and T.C. Lubensky, Phys. Rev. A 14, 2307 (1976).

[13] A. Linhananta and D.E. Sullivan, Phys. Rev. A 44, 8189 (1991).

[14] R.F. Kayser and H.J. Raveché, Phys. Rev. A 17, 2067 (1978).
[15] J. Herzfeld, A.E. Berger, and J.W. Wingate, Macromolecules 17, 1718 (1984).

[16] H.N.W. Lekkerkerker, Ph. Coulon, R. Van Der Haegen, and R. Deblieck, J. Chem. Phys. 80, 3427 (1984).

[17] A. Poniewierski, Phys. Rev. A 45, 5605 (1992).

[18] J.D. Parson, Phys. Rev. A 19, 1225 (1979).

[19] S.D. Lee, J. Chem. Phys. 87, 4972 (1987).

[20] Our calculations on the Zwanzig model (discrete orientations) [21] show that this coupling eliminates the predicted tricritical point on the $N-\mathrm{Sm}-A$ transition line $[8,9,22]$, turning the transition into a continuous one for all $\kappa$. However, predicting the nature of the $N-\mathrm{Sm}-A$ transition seems to be a very subtle problem [17].

[21] R. Zwanzig, J. Chem. Phys. 39, 1714 (1963).

[22] M. Masahito, H. Nakano, and H. Kimura, J. Phys. Soc. Jpn. 47, 740 (1979).

[23] F.P. Booy and A.G. Fowler, Int. J. Biol. Macromol. 7, 327 (1985). 


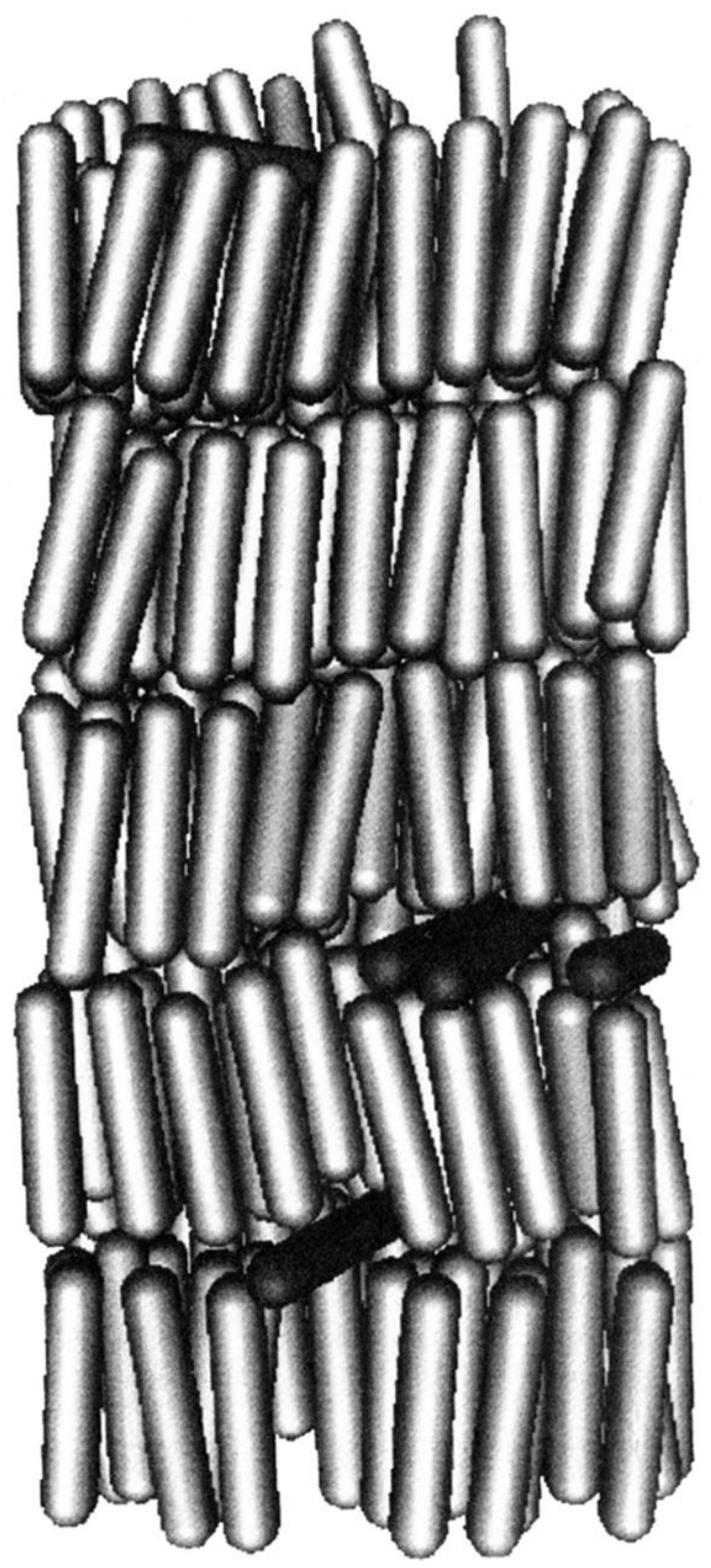

FIG. 3. Typical equilibrated configuration of the Sm-A phase of spherocylinders with $\kappa=3.8$ at $\eta=0.527$. We highlighted the transverse interlayer particles. The apparent alignment of these particles is accidental; statistically there is no preferred in-plane direction. From the ODF in Fig. 2(b) one would expect to see more "parallel" than "transverse" particles in between the layers in a single snapshot. The low density of particles in between the layers, however, prevented us from finding a single snapshot conveniently visualizing both possible orientations at the same time. 